Published in final edited form as:

J Am Chem Soc. 2005 October 12; 127(40): 13766-13767.

\title{
Investigating Bacterial N-Linked Glycosylation: Synthesis and Glycosyl Acceptor Activity of the Undecaprenyl Pyrophosphate- Linked Bacillosamine
}

\author{
Eranthie Weerapana, Kerney Jebrell Glover, Mark M. Chen, and Barbara Imperiali* \\ Departments of Chemistry and Biology, Massachusetts Institute of Technology, Cambridge, \\ Massachusetts 02139
}

\begin{abstract}
Asparagine-linked protein glycosylation has long been considered a unique feature of the eukaryotic kingdom; however, recent studies have revealed the presence of $\mathrm{N}$-linked glycoproteins in Campylobacter jejuni, a gram-negative bacterium that is involved in human gastroenteritis. ${ }^{1}$ The process of $\mathrm{N}$-linked glycosylation in $C$. jejuni displays significant similarities to the analogous process in eukaryotes. ${ }^{2}$ A series of glycosyltransferases act sequentially to assemble a glycan donor on a polyisoprenyl carrier (undecaprenyl pyrophosphate, Und-PP), which is transferred to the asparagine side chain of proteins at the $\mathrm{N}-\mathrm{X}-\mathrm{S} / \mathrm{T}$ consensus sequence. ${ }^{3}$ In $C$. jejuni, the glycan donor has been identified as the UndPP-linked heptasaccharide GalNAc- $\alpha 1,4$ - GalNAc- $\alpha 1,4-(\mathrm{Glc} \beta 1,3)-G a l N A c-\alpha 1,4-G a l-N A c-$ $\alpha 1,4-G a l N A c-\alpha 1,3-B a c-\alpha 1, P P-U n d ~(\mathbf{1})$, where Bac is the unusual sugar bacillosamine (2,4diacetamido-2,4,6-trideoxyglu-cose) that is only found in specific bacterial systems (Figure 1). ${ }^{4}$ Bioinformatic and mutagenesis data suggest that the five glycosyltransferases (Pgl C, A, $\mathrm{J}, \mathrm{H}$, and I located in the $p g l$ gene cluster) are responsible for the biosynthesis of the heptasaccharide. ${ }^{5}$
\end{abstract}

To study these glycosyltransferases in vitro, large quantities of highly pure undecaprenyllinked glycan substrates are essential. These polyisoprene-linked compounds are only present in very small quantities, making purification from native sources an unrealistic endeavor. Furthermore, this is complicated by the fact that these strains of bacteria are extremely pathogenic, thus requiring specialized handling. Employing chemical synthesis, we can access the desired milligram quantities of the Und-PP-linked glycans for biochemical studies of the glycosyltransferases. Here we report the synthesis of Und-PP-Bac (2) (Scheme 1), a key intermediate in the Pgl pathway, and initial efforts to investigate the activity of the Pgl glycosyltransferases in vitro using this synthetic substrate.

The synthesis of $\mathbf{2}$ involves the coupling of undecaprenyl phosphate to bacillosamine 6 phosphate $\mathbf{3}$, which is synthesized from benzyl 2 -acetamido-2-deoxy- $\beta$-р-galactopyranoside 4. ${ }^{7}$ The $\mathrm{C}-4$ azido functionality was installed by a selective benzoylation of C-3 and C-6 followed by triflation and subsequent displacement with sodium azide at C-4 resulting in intermediate 5 . To deoxygenate at C-6, the iodo functionality was introduced to afford $\mathbf{6}$, which was reduced concurrently with the C-4 azide by catalytic hydrogenation. Subsequent acetylation of both the amino and hydroxyl functionalities resulted in intermediate 7. At this stage, to improve the solubility properties of the intermediate and render it compatible with subsequent transformations, it was necessary to exchange the C-3 acetyl-protecting group for a benzoyl group to afford $\mathbf{8}$. Deprotection of the anomeric hydroxyl followed by

E-mail:imper@mit.edu.

Supporting Information Available: Experimental procedures and product characterization for all new compounds synthesized. Cloning and expression of PglA and procedures for assaying activity. This material is available free of charge via the Internet at http://pubs.acs.org. 
phosphorylation with tetrabenzyl pyrophosphate afforded the phosphorylated bacillosamine derivative 3. Phosphorylation with tetrabenzyl pyrophosphate proceeds with $>16: 1$ selectivity in favor of the $\alpha$-phosphate. However, upon deprotection, a minor $(<15 \%)$ impurity in the product is observed, which cannot be separated by chromotography. The minor product may be due to epimerization at the anomeric center.

Undecaprenyl phosphate was synthesized from undecaprenol using phosphoramidite chemistry as previously reported. ${ }^{8}$ The coupling to bacillosamine phosphate $\mathbf{3}$ was effected using activation with 1,1'-carbonyldiimidazole. In the final step, the C-3 benzoyl ester was removed with sodium methoxide, resulting in the first chemical synthesis of Und-PP-Bac (2).

In vivo mutagenesis studies have shown that $\mathrm{PglA}$ is the glycosyltransferase that catalyzes the transfer of a GalNAc residue from UDP-GalNAc to 2 to form GalNAc- $\alpha 1,3-B a c-\alpha 1-P P-U n d$ (9) (Figure 2a). ${ }^{9}$ To validate PglA activity in vitro, Und-PP-Bac (2) was used as the cosubstrate with UDP-GalNAc for purified PglA, which was cloned and overexpressed in Escherichia coli. The preliminary enzyme activity assay involved monitoring the transfer of radiolabeled GalNAc from aqueous-soluble UDP- $\left[{ }^{3} \mathrm{H}\right]$-GalNAc to organic-soluble $\left[{ }^{3} \mathrm{H}\right]$-GalNAc-Bac-PPUnd in the presence of purified PglA (Figure 2b). The significant increase in radioactivity in the organic extract upon addition of $\mathbf{2}$ confirms that PglA accepts this synthetic substrate very efficiently.

The disaccharide product from this enzymatic reaction was characterized by acidic hydrolysis of the saccharide from the undecaprenyl pyrophosphate carrier to yield $\mathbf{1 0}$, followed by labeling of the reducing terminus with 2-aminobenzamide ( $2 \mathrm{AB})$ via a reductive amination to afford 11. The resulting $2 \mathrm{AB}$-labeled disaccharide $\mathbf{1 1}$ was isolated on a GlykoSepN normal-phase column and analyzed by MALDI-MS (Figure 3).

The synthetic route outlined herein provides access to milligram quantities of Und-PP-Bac, which is the first membrane-associated substrate in the $C$. jejuni $\mathrm{N}$-linked protein glycosylation pathway. The availability of this substrate has further enabled validation of the enzymatic activity of the glycosyltransferase PglA, revealing that Und-PP-Bac serves as the glycosyl acceptor with UDP- $N$-acetyl galactosamine as the glycosyl donor in the enzyme-catalyzed transformation. The disaccharide obtained from the PglA reaction has recently been used to investigate the glycosyltransferases $\mathrm{Pgl} \mathrm{J}, \mathrm{H}$, and I, resulting in the chemoenzymatic synthesis of the Und-PP-heptasaccharide $1 .{ }^{10}$ Access to Und-PP-Bac removes a significant obstacle in the study of bacterial N-linked glycosylation and paves the way for biophysical and biochemical analysis of the process. These studies will provide an important foundation for studies targeted at understanding the more complex, yet analogous, process of $\mathrm{N}$-linked glycosylation in eukaryotic systems.

\section{Acknowledgements}

This research was supported by the NIH (GM39334) and postdoctoral fellowship (GM65699) to K.J.G.

\section{References}

1. a Szymanski CM, Yao R, Ewing CP, Trust TJ, Guerry P. Mol Microbiol 1999;32:1022-1030. [PubMed: 10361304] b Szymanski CM, Wren BW. Nat Rev Microbiol 2005;3:225-237. [PubMed: 15738950]

2. Burda P, Aebi M. Biochim Biophys Acta 1999;1426:239-257. [PubMed: 9878760]

3. Nita-Lazar M, Wacker M, Schegg B, Amber S, Aebi M. Glycobiology 2005;15:1957-1964.

4. Young MN, Brisson JR, Kelly J, Watson DC, Tessier L, Lanthier PH, Jarrell HC, Cadotte N, St Michael F, Aberg E, Szymanski CM. J Biol Chem 2002;277:42530-42539. [PubMed: 12186869]

5. Wacker M, Linton D, Hitchen PG, Nita-Lazar M, Haslam SM, North SJ, Panico M, Morris HR, Dell A, Wren BW, Aebi M. Science 2002;298:1790-1793. [PubMed: 12459590] 
6. Previous syntheses of bacillosamine derivatives: (a) LievAHildesheimJZehaviUSharonNCarbohydr Res197433217227 b Bundle DR, Josephson S. Can J Chem 1980;58:2679-2685.

7. Matta KL, Johnson EA, Barlow JJ. Carbohydr Res 1973;26:215-218.

8. Synthesis of undecaprenyl phosphate: (a) BranchCLBurtonGMossSFSynth Commun 19992926392644 b Ye XY, Lo MC, Brunner L, Walker D, Kahne D, Walker S. J Am Chem Soc 2001;123:3155-3156. [PubMed: 11457035]

9. Linton D, Dorrel N, Hitchen PG, Amber S, Karlysev AV, Morris HR, Dell A, Valvano MA, Aebi M, Wren BW. Mol Microbiol 2005;55:1695-1703. [PubMed: 15752194]

10. Glover, K. J.; Weerapana, E.; Imperiali, B. Proc. Natl. Acad. Sci. U.S.A., in press. 


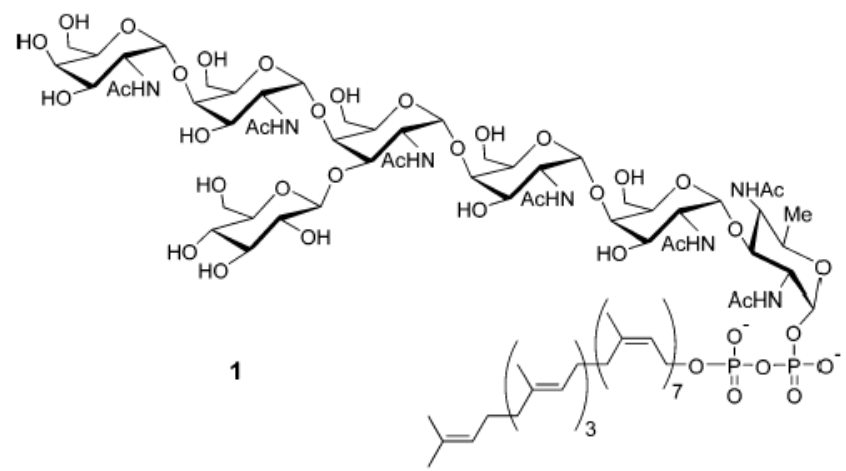

Figure 1.

Structure of the undecaprenyl-linked heptasaccharide. 
(a)

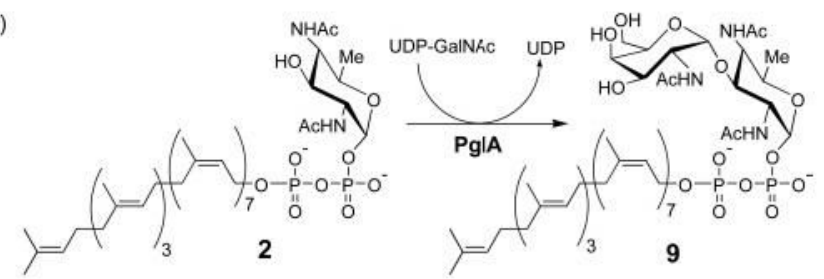

(b)
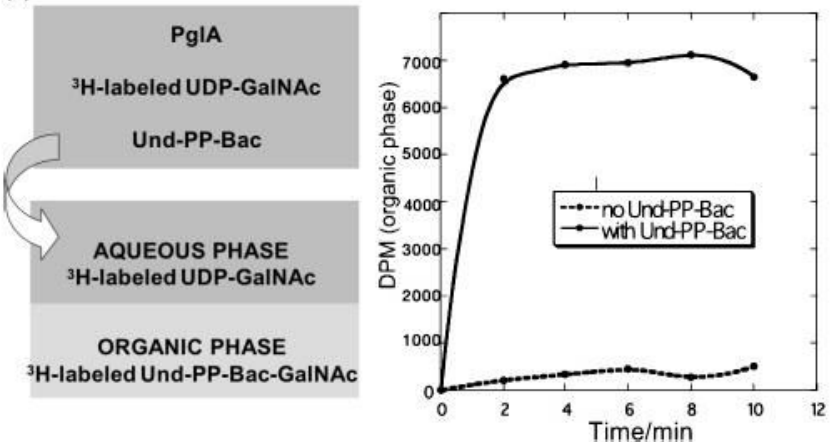

Figure 2.

(a) Reaction catalyzed by PglA. (b) Radioactive assay for PglA activity with Und-PP-Bac, 2. DPM (disintegrations per minute) denotes radioactive counts in the organic phase. 

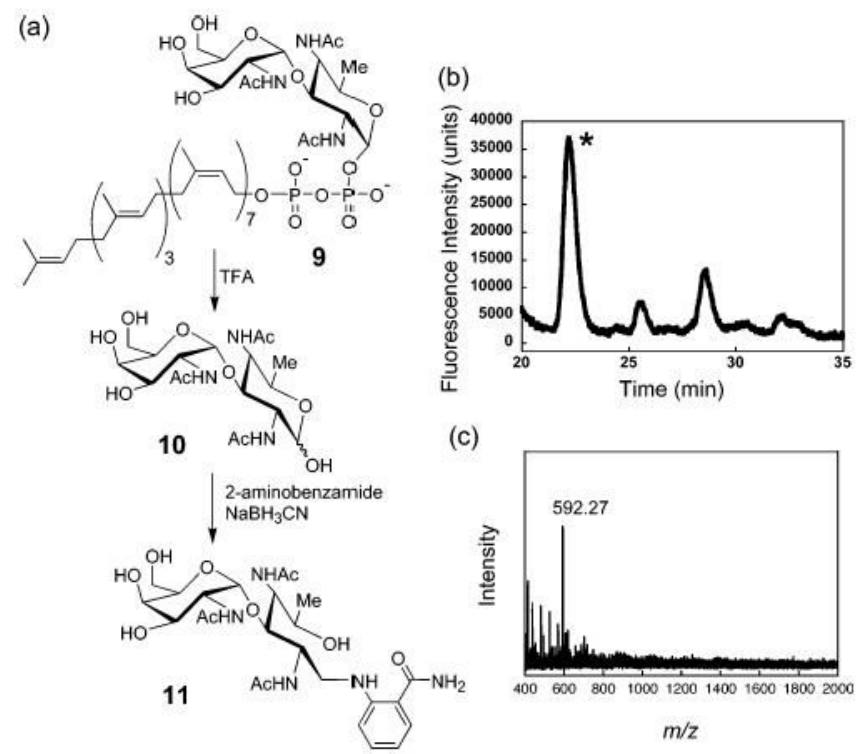

(c)

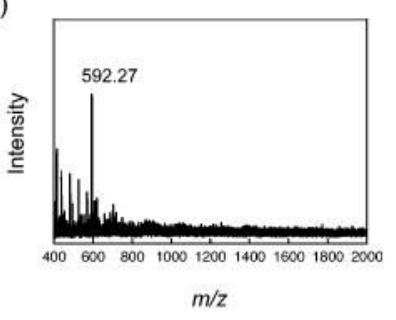

Figure 3.

(a) Hydrolysis and 2-aminobenzamide labeling. (b) HPLC trace of 2AB-labeled GalNAc-Bac (fluorescence detection). * Denotes major product peak. (c) MALDI-MS of disaccharide (peak at 592.27 denotes sodium adduct of expected peak). 


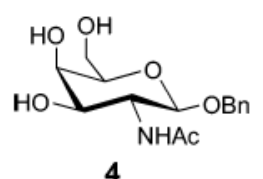

4

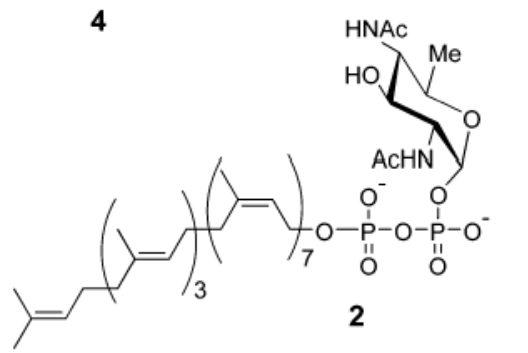

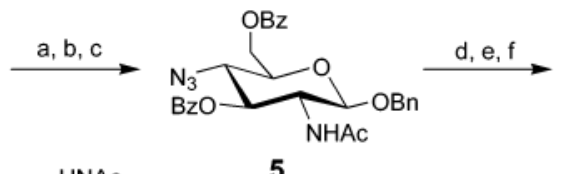

5

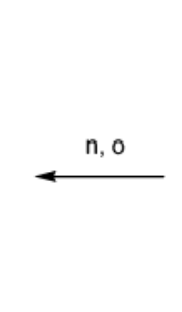

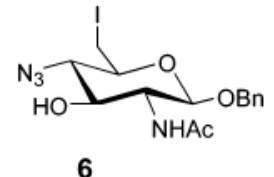

6

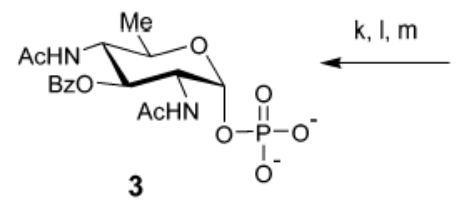

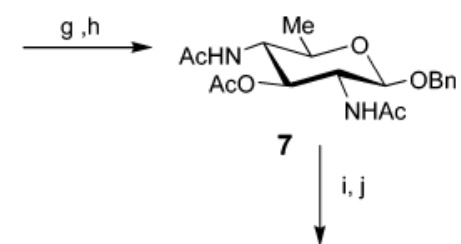

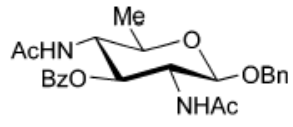

8

Scheme 1.

Synthesis of Und-PP-Bac ${ }^{a}$

${ }^{a}$ Reagents and conditions: (a) benzoyl chloride, pyridine, $-40 \rightarrow 0{ }^{\circ} \mathrm{C}, 70 \%$; (b) $\mathrm{Tf}_{2} \mathrm{O}$, $\mathrm{CH}_{2} \mathrm{Cl}_{2}$ /pyridine, $0{ }^{\circ} \mathrm{C} \rightarrow \mathrm{rt}$; (c) $\mathrm{NaN}_{3}$, DMF, rt, $62 \%$ over two steps; (d) NaOMe, MeOH, rt, $80 \%$; (e) TsCl, pyridine, $0{ }^{\circ} \mathrm{C} \rightarrow \mathrm{rt}, 75 \%$; (f) NaI, $\mathrm{MeCN}, 80{ }^{\circ} \mathrm{C}, 70 \%$; (g) $\mathrm{H}_{2}, \mathrm{Pd}(\mathrm{OH})_{2} / \mathrm{C}$, DIPEA, $\mathrm{MeOH}, 32{ }^{\circ} \mathrm{C}$; (h) $\mathrm{Ac}_{2} \mathrm{O}$, pyridine, rt, $65 \%$ over two steps; (i) $\mathrm{NaOMe}, \mathrm{MeOH}, 90 \%$; (j) $\mathrm{BzCl}$, pyridine, $60 \%$; (k) $\mathrm{H}_{2}, \mathrm{Pd}(\mathrm{OH})_{2} / \mathrm{C}, \mathrm{MeOH}, 32{ }^{\circ} \mathrm{C}, 86 \%$; (l) LiHMDS, $-68{ }^{\circ} \mathrm{C}$; then $\left[(\mathrm{BnO})_{2} \mathrm{P}(\mathrm{O})\right]_{2} \mathrm{O},-68 \rightarrow 0{ }^{\circ} \mathrm{C}, 50 \%$; (m) $\mathrm{H}_{2}, \mathrm{Pd} / \mathrm{C}, \mathrm{MeOH}, 99 \%$; (n) carbonyl diimidazole, DMF; then undecaprenyl phosphate, $50 \%$; (o) $\mathrm{NaOMe}, \mathrm{MeOH}, 99 \%$. 